\title{
ceRNA analysis of SARS-CoV-2
}

\author{
Walter Arancio ${ }^{1,2,3}$
}

Received: 22 June 2020 / Accepted: 14 September 2020 / Published online: 17 November 2020

(c) Springer-Verlag GmbH Austria, part of Springer Nature 2020

\begin{abstract}
Viral RNAs can perturb the miRNA regulatory network, competing with host RNAs as part of their infective process. An in silico competing endogenous RNA (ceRNA) analysis has been carried on SARS-CoV-2. The results suggest that, in humans, the decrease of microRNA activity caused by viral RNAs can lead to a perturbation of vesicle trafficking and the inflammatory response, in particular by enhancing KLF10 activity. The results suggest also that, during the study of the mechanics of viral infections, it could be of general interest to investigate the competition of viral RNA with cellular transcripts for shared microRNAs.
\end{abstract}

The global outbreak of a novel betacoronavirus named SARS-CoV-2 (causing COVID-19) has posed a global health emergency. Although SARS-CoV-2 usually causes mild symptoms, some patients develop fatal complications including severe pneumonia and acute respiratory distress syndrome. At present, there are few therapeutic procedures (the majority of them are being used on a trial basis) and no preventive vaccines for COVID-19. Due to its novelty, the detailed pathophysiological mechanisms of COVID-19 is largely unknown, and our knowledge is mainly inferred from previous studies on other coronaviruses, which, however, cannot give a full explanation of the inflammatory response that can lead in turn to severe pneumonia and death [1].

Viral infection is a multilayered event that can be simplified in a step-by-step process beginning with attachment to a specific membrane receptor of the target cell and cell penetration, uncoating of the viral particle, hijacking of the

Handling Editor: Akbar Dastjerdi.

Electronic supplementary material The online version of this article (https://doi.org/10.1007/s00705-020-04856-4) contains supplementary material, which is available to authorized users.

Walter Arancio

walter.arancio@gmail.com; walter.arancio@unipa.it

1 University of Palermo, Viale delle Scienze, 90127 Palermo, Italy

2 Scuola “Borgese XXVII Maggio", Piazza Contardo Ferrini, 13, 90146 Palermo, Italy

3 Advanced data analysis group, Fondazione Ri.MED, Via Bandiera 11, 90133 Palermo, Italy cellular program for viral replication, assembly of viral particles, and release of mature infectious forms of the virus. Each of these steps is dissected by researchers in order to understand the mechanics of viral infection and to design potential therapies interfering with key viral mechanisms.

An often-overlooked aspect of viral infection regards the non-coding role of viral RNAs within infected cells, more so if considering the significant abundance of viral transcripts during an ongoing infection. Competing endogenous RNA (or ceRNA) analysis allows predictions to be made about RNAs that can regulate other RNA transcripts by competing for shared microRNAs (miRNAs), which are key players of cellular homeostasis in eukaryotes with recognized regulatory roles that are often used in diagnosis, prognosis, monitoring, and therapy [2-4]. ceRNA analyses have been able to give plausible explanations of several biological phenomena that would otherwise be difficult to interpret, although caution is always recommended in understanding the true meaning of the analyses, and strict validation procedures are always required [5-8]. The high variability of the symptoms and prognosis of COVID-19 patients suggests that there are many aspects of SARS-CoV-2 infection that still need to be clarified. Therefore a ceRNA analysis on how SARS-CoV-2 infection can perturb the regulatory network of interactions between miRNAs and messenger RNAs was carried out.

In order to perform an in silico ceRNA analysis to identify host gene transcripts that might be perturbed by SARSCoV-2 RNAs, the NCBI Reference Sequence NC_045512.2 of SARS-CoV-2 was analyzed using miRDB (https://mirdb .org), an online database for miRNA target prediction and functional annotation $[9,10]$. By custom target prediction, 


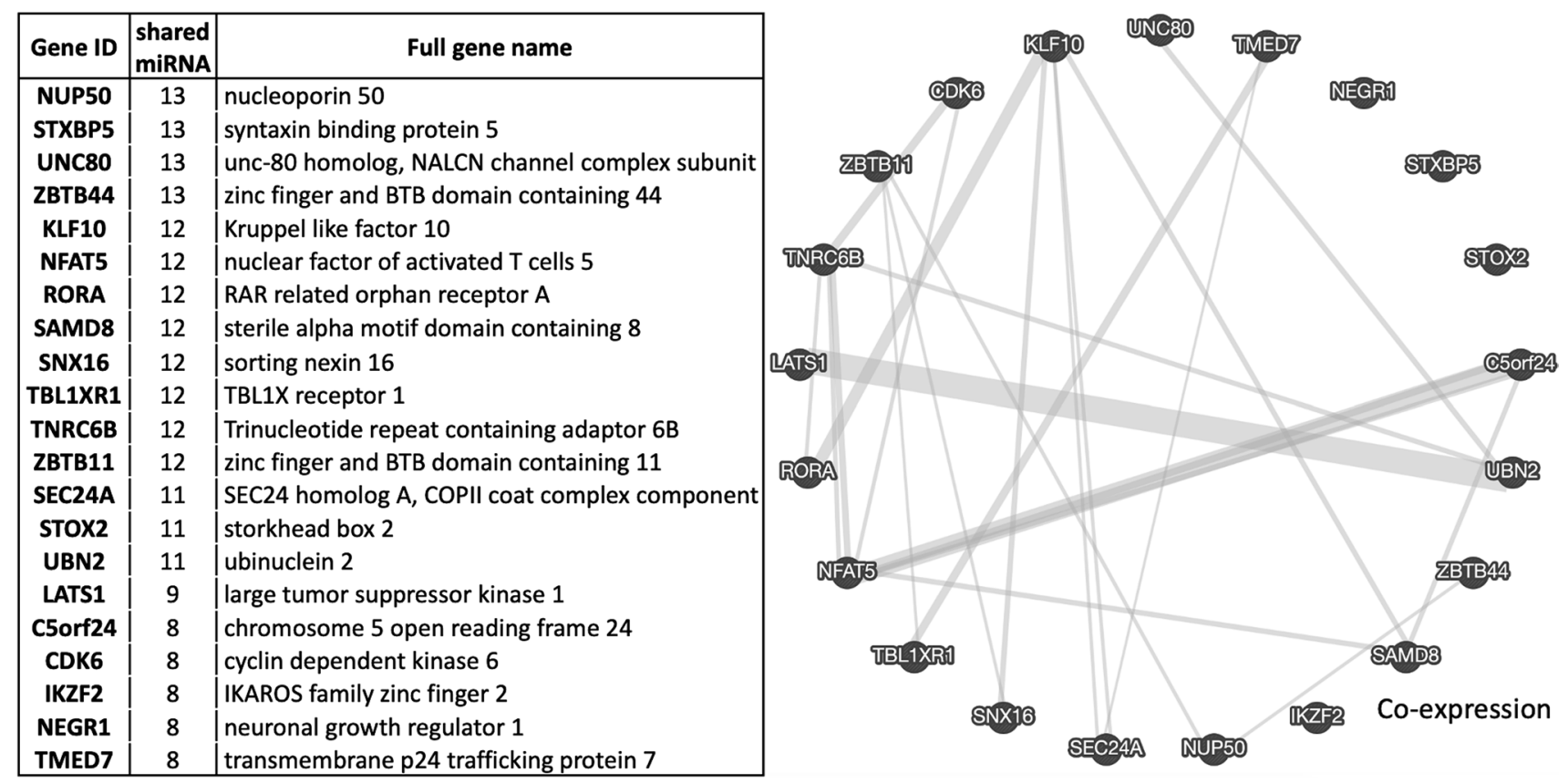

Fig. 1 Genes that code for competing endogenous RNAs for SARS-CoV-2 transcripts are usually co-expressed.

a panel of miRNA sequences able to recognize the SARS$\mathrm{CoV}-2$ sequence was collected. Only targets with a very high score (95-100) were retained for further analyses. Human gene targets for each miRNA isolated were predicted with very high stringency (including only functional miRNAs of the FuncMir collection with a target prediction score of 95 or above and excluding miRNAs with more than 5000 predicted targets in the genome). The genes were then ordered according to the number of miRNAs that recognized each gene transcript: the higher the number, the higher the probability that a particular gene can code for a competing endogenous RNA for SARS-CoV-2 RNAs. The rationale and a protocol were the same as in other studies $[5,6]$ with minor modifications. The detailed results of the analysis are reported in Supplementary material 1. Network analysis was performed using the Genemania tool (https://genemania.org) [11]. The 2-tailed Student's $t$-test was performed in Excel. A heat map was drawn using Heatmapper [12], with the average linkage clustering method and Manhattan distance measurement.

The ceRNA analysis was performed twice, on both the positive and negative strand of the SARS-CoV-2 sequences, but only the positive strand yielded significant results (as detailed in Supplementary material 1), suggesting that the positive strand might indeed be able to regulate endogenous RNAs competing for shared miRNA and that the results are not just only a procedural artefact. The results were not weighted for the relative abundance of transcripts.

A total of 25 functional miRNAs satisfied all of the stringent requirements described previously, and each human gene transcript was recognized by no more than 13 miRNAs. Only 21 genes were recognized by eight or more miRNAs, and those genes were thus considered putative ceRNAs and retained for further network analysis. The selected genes were usually co-expressed, suggesting that they could be co-regulated by a miRNA network. The results are reported in Fig. 1.

Interestingly, the selected genes are involved in cell cycle regulation (CDK6 and LATS1) and intracellular vesicle trafficking (SEC24A, SNX16, STXBP5, TMED7), which is essential for the viral life cycle, together with inflammatory and immune responses, which are key aspects of the pathological mechanisms of COVID-19 and the host response. In detail, KLF10 is involved in the antiviral immune response [13], NFAT5 limits infection-induced type I interferon responses [14], RORA is a negative regulator of the inflammatory response [15], and IKZF2, also known as Helios, codes for a hematopoietic-specific transcription factor that is essential for regulatory T-cell development [16].

Although the analysis was performed using very stringent criteria, several genes that are potentially involved in SARSCoV-2 infection (vesicle trafficking) and the pathophysiological mechanisms of COVID-19 (inflammatory and immune 
response) were identified. A number of studies have shown that the immunological and inflammatory response is crucial in the pathological mechanism of COVID-19 [17-19] and might be associated with pediatric multi-system inflammatory syndrome [20] and cytokine storm [21]. It has also been reported that the severity of COVID-19 might be influenced by host miRNAs in elderly patients [22], who tend to exhibit chronic inflammation (inflammaging) and represent the vast majority of COVID-19 victims [1].

In order to validate the analysis, datasets from differential expression analysis based on RNA sequencing by BlancoMelo and colleagues were used [23]. An extract of the analysis regarding only the ceRNA genes examined here can be found in Supplementary material 2.

A comparison of epithelial lung cancer cell lines (Calu-3 [24] and A549 cells made permissive to the infection) infected with SARS-CoV-2 with uninfected cells has pointed to several putative ceRNA genes that are upregulated more than twofold after SARS-CoV-2 infection (i.e., KLF10, NFAT5, RORA, SAMD8, SNX16, SEC24A, and LATS1), consistent with the original ceRNA model [7]. In particular, KLF10 expression is upregulated more than sixfold after SARS-CoV-2 infection in Calu- 3 cells and more than eightfold in A549 cells expressing the viral receptor ACE2. Interestingly, A549 cells, which naturally lack the receptor, showed only a modest increase in KLF10 transcripts. Moreover, the increase in KLF10 transcripts was not hampered by the addition of ruxolitinib, a JAK1 and JAK2 kinase inhibitor, suggesting that the increase in $K L F 10$ transcripts is independent of IFN-I and -III signaling [23].

However, when normal human bronchial epithelial cells were infected with SARS-CoV-2, no significant differential expression of ceRNA genes was observed in comparison with uninfected controls. When ferrets were used as an animal model of the disease, the results were highly variable. Transcription of KLF10 increased at the beginning of the observation period and then progressively decreased in the infected upper respiratory cell populations in comparison with uninfected controls, while in the tracheas there was only a small increase caused by the infection. However, in the trachea, the vast majority of the other ceRNA genes are upregulated by SARS-CoV-2 infection. Finally, when comparing post-mortem lung tissues of COVID-19 patients and controls, a generalized downregulation of ceRNA genes can be observed, with the exception of the highly expressed NFAT5. This is probably due to the inflammatory infiltrate, and possibly other factors.

In order to test if the expression of the ceRNA genes are altered in immune cells upon SARS-CoV-2 infection, two independent panels of PBMCs from patients and controls were used (NCBI accession numbers GSE155106 and GSE152418). Interestingly, in those cells, the only gene that was both highly expressed and showed an increase in its expression upon infection was KLF10. In GSE155106, $K L F 10$ showed an almost threefold increase in expression in human PBMC-derived dendritic cells and macrophages upon SARS-CoV-2 infection, while in GSE152418, its expression increased slightly (1.2 times) in PBMCs of a group of 17 COVID-19 subjects in comparison with 17 healthy controls, but this increase was statistically significant $(p<0,05)$. An extract of the analysis is presented in Supplementary material 3 , and the heat map of expression of the ceRNA genes present in GSE152418 is presented in Supplementary material 4.

Although KLF1O is usually recognized as an antiinflammatory gene, its role is far from straightforward [13, 25-27]. While KLF10 is involved in the antiviral immune response [13], it has been suggested to play a role in pathological airway remodeling [25] and fibrosis in other organs [26], probably during the TGF beta response [27]. It is noteworthy that, although no commercial drugs are available that target $K L F 10$, small molecule-inhibitors have been already identified [28].

The interpretation of these data is far from straightforward, but a possible explanation is that competing endogenous RNAs can play a role at the beginning of SARS-CoV-2 infection in specific tissues and under specific conditions and that their role is less crucial after tissue remodelling due to COVID-19 disease, highlighting the cell-intrinsic role of these ceRNAs. The significant increase in KLF10 transcripts in immune cells of infected patients and in a subset of permissive cells upon infection suggest a possible role of this gene in the etiopathogenesis of COVID-19 upon SARS-Cov-2 infection that is worth further investigation.

It is possible that the abundant viral RNAs present in the cell during infection may specifically perturb the miRNA regulatory network, competing with host RNAs as part of their infectious process. This could plausibly be a widespread phenomenon that is not limited to SARS-CoV-2 infection. The results suggest that SARS-CoV-2 RNAs can lead to specific perturbations in vesicle trafficking and the inflammatory response, in particular by enhancing KLF10 activity.

Funding Not applicable.

Availability of data and material Data are derived from public domain resources.

Code availability Codes are from public domain resources.

\section{Compliance with ethical standards}

Conflict of interest The author has no competing interests to declare. 


\section{References}

1. Sohrabi C, Alsafi Z, O’Neill N, Khan M, Kerwan A, Al-Jabir A, Iosifidis C, Agha R (2020) World Health Organization declares global emergency: a review of the 2019 novel coronavirus (COVID-19). Int J Surg. 76:71-76. https://doi.org/10.1016/j. ijsu.2020.02.034

2. Arancio W, Amato MC, Magliozzo M, Pizzolanti G, Vesco R, Giordano C (2018) Serum miRNAs in women affected by hyperandrogenic polycystic ovary syndrome: the potential role of miR-155 as a biomarker for monitoring the estroprogestinic treatment. Gynecol Endocrinol. 34(8):704-708. https://doi. org/10.1080/09513590.2018.1428299

3. Cheng G (2015) Circulating miRNAs: roles in cancer diagnosis prognosis and therapy. Adv Drug Deliv Rev. 81:75-93. https:// doi.org/10.1016/j.addr.2014.09.001

4. Kong YW, Ferland-McCollough D, Jackson TJ, Bushell M (2012) microRNAs in cancer management. Lancet Oncol. 13(6):e249e258. https://doi.org/10.1016/S1470-2045(12)70073-6

5. Arancio W, Carina V, Pizzolanti G, Tomasello L, Pitrone M, Baiamonte C, Amato MC, Giordano C (2015) Anaplastic thyroid carcinoma: a ceRNA analysis pointed to a crosstalk between SOX2, TP53, and microRNA biogenesis. Int J Endocrinol. 2015:439370. https://doi.org/10.1155/2015/439370

6. Arancio W, Genovese SI, Bongiovanni L, Tripodo C (2015) A ceRNA approach may unveil unexpected contributors to deletion syndromes, the model of 5q- syndrome. Oncoscience 2(10):872-879

7. Salmena L, Poliseno L, Tay Y, Kats L, Pandolfi PP (2011) A ceRNA hypothesis: the Rosetta stone of a hidden RNA language? Cell 146(3):353-358. https://doi.org/10.1016/j.cell.2011.07.014

8. Tay Y, Rinn J, Pandolfi PP (2014) The multilayered complexity of ceRNA crosstalk and competition. Nature 505(7483):344-352. https://doi.org/10.1038/nature12986

9. Chen Y, Wang X (2020) miRDB: an online database for prediction of functional microRNA targets. Nucleic Acids Res 48(D1):D127-D131

10. Liu W, Wang X (2019) Prediction of functional microRNA targets by integrative modeling of microRNA binding and target expression data. Genome Biol. 20(1):18. https://doi.org/10.1186/s1305 9-019-1629-z

11. Warde-Farley D, Donaldson SL, Comes O, Zuberi K, Badrawi R, Chao P, Franz M, Grouios C, Kazi F, Lopes CT, Maitland A, Mostafavi S, Montojo J, Shao Q, Wright G, Bader GD, Morris Q (2010) The GeneMANIA prediction server: biological network integration for gene prioritization and predicting gene function. Nucleic Acids Res. 38(Web Server issue):W214-W220. https:// doi.org/10.1093/nar/gkq537

12. Babicki S, Arndt D, Marcu A, Liang Y, Grant JR, Maciejewski A, Wishart DS (2016) Heatmapper: web-enabled heat mapping for all. Nucleic Acids Res. 44(W1):W147-W153. https://doi. org/10.1093/nar/gkw419

13. Papadakis KA, Krempski J, Reiter J, Svingen P, Xiong Y, Sarmento OF, Huseby A, Johnson AJ, Lomberk GA, Urrutia RA, Faubion WA (2015) Krüppel-like factor KLF10 regulates transforming growth factor receptor II expression and TGF- $\beta$ signaling in CD8+ T lymphocytes. Am J Physiol Cell Physiol. 308(5):C362-C371. https://doi.org/10.1152/ajpcell.00262.2014

14. Huerga Encabo H, Traveset L, Argilaguet J, Angulo A, NistalVillán E, Jaiswal R, Escalante CR, Gekas C, Meyerhans A, Aramburu J, López-Rodríguez C (2020) The transcription factor
NFAT5 limits infection-induced type I interferon responses. J Exp Med. 217(3):e20190449. https://doi.org/10.1084/jem.20190449

15. Delerive P, Monté D, Dubois G, Trottein F, Fruchart-Najib J, Mariani J, Fruchart JC, Staels B (2001) The orphan nuclear receptor ROR alpha is a negative regulator of the inflammatory response. EMBO Rep. 2(1):42-48

16. Thornton AM, Shevach EM (2019) Helios: still behind the clouds. Immunology 158(3):161-170. https://doi.org/10.1111/imm.13115

17. Fu Y, Cheng Y, Wu Y (2020) Understanding SARS-CoV-2-mediated inflammatory responses: from mechanisms to potential therapeutic tools. Virol Sin. https://doi.org/10.1007/s12250-020-00207 $-4$

18. Tay MZ, Poh CM, Rénia L, MacAry PA, Ng LFP (2020) The trinity of COVID-19: immunity, inflammation and intervention. Nat Rev Immunol. 20(6):363-374. https://doi.org/10.1038/s4157 7-020-0311-8

19. Prete M, Favoino E, Catacchio G, Racanelli V, Perosa F (2020) SARS-CoV-2 inflammatory syndrome. Clinical features and rationale for immunological treatment. Int J Mol Sci. 21(9):3377. https://doi.org/10.3390/ijms21093377 (Published 2020 May 10)

20. Deza Leon MP, Redzepi A, McGrath E et al (2020) COVID-19 associated pediatric multi-system inflammatory syndrome. J Pediatric Infect Dis Soc. https://doi.org/10.1093/jpids/piaa061

21. Ye Q, Wang B, Mao J (2020) The pathogenesis and treatment of the 'Cytokine Storm' in COVID-19. J Infect. 80(6):607-613. https ://doi.org/10.1016/j.jinf.2020.03.037

22. Fulzele S, Sahay B, Yusufu I, Lee TJ, Sharma A, Kolhe R, Isales CM (2020) COVID-19 virulence in aged patients might be impacted by the host cellular microRNAs abundance/ profile. Aging Dis 11(3):509-522. https://doi.org/10.14336/ AD. 2020.0428

23. Blanco-Melo D, Nilsson-Payant BE, Liu WC, Uhl S, Hoagland D, Møller R, Jordan TX, Oishi K, Panis M, Sachs D, Wang TT, Schwartz RE, Lim JK, Albrecht RA, tenOever BR (2020) Imbalanced host response to SARS-CoV-2 drives development of COVID-19. Cell 181(5):1036-1045.e9. https://doi.org/10.1016/j. cell.2020.04.026

24. Zhu Y, Chidekel A, Shaffer TH (2010) Cultured human airway epithelial cells (calu-3): a model of human respiratory function, structure, and inflammatory responses. Crit Care Res Pract 2010:394578. https://doi.org/10.1155/2010/394578

25. Fueki N, Sagara H, Akimoto K et al (2007) Interleukin-10 regulates transforming growth factor-beta signaling in cultured human bronchial epithelial cells. Respiration. 74(4):454-459. https://doi. org/10.1159/000101057

26. Kim JK, Lee KS, Chang HY, Lee WK, Lee JI (2014) Progression of diet induced nonalcoholic steatohepatitis is accompanied by increased expression of Kruppel-like-factor 10 in mice. J Transl Med. 12:186. https://doi.org/10.1186/1479-5876-12-186

27. Subramaniam M, Hawse JR, Rajamannan NM, Ingle JN, Spelsberg TC (2010) Functional role of KLF10 in multiple disease processes. BioFactors 36(1):8-18. https://doi.org/10.1002/biof.67

28. Khedkar SA, Sun X, Rigby AC, Feinberg MW (2015) Discovery of small molecule inhibitors to Krüppel-like factor 10 (KLF10): implications for modulation of $\mathrm{T}$ regulatory cell differentiation. $\mathrm{J}$ Med Chem 58(3):1466-1478. https://doi.org/10.1021/jm5018187

Publisher's Note Springer Nature remains neutral with regard to jurisdictional claims in published maps and institutional affiliations. 